\title{
RNA-Mediated Crop Improvement
}

\section{Basel Khraiwesh}

Plant Stress Genomics Research Center, King Abdullah University of Science and Technology, Thuwal 23955-6900, Kingdom of Saudi Arabia

Indeed, one of the more recent discoveries in molecular biology of non-coding RNAs is RNA interference (RNAi). RNAi is a method of blocking gene function by inserting short sequences of ribonucleic acid (RNA) that match part of the target gene's sequence, thus no proteins are produced. Since Science named it as "Breakthrough of the Year" and Fortune magazine hailed it as "Biotech's Billion Dollar Breakthrough". RNAi is a mechanism regulating gene transcript levels by either transcriptional gene silencing (TGS) or by posttranscriptional gene silencing (PTGS), which acts in genome maintenance and the regulation of development [1]. Since the discovery of RNAi in Caenorhabditis elegans extensive studies have been performed focusing on the different aspects of RNAi. In particular, the elucidation of the essential components of RNAi pathways has advanced extensively. RNAi has been discovered in a wide range of organisms from plants and fungi to insects and mammals suggesting that it arose early in the evolution of multicellular organisms.

RNAi has the potential to become a powerful therapeutic approach toward targeted and personalized medicine. In medical research, RNAi is on the way to becoming an important tool to treat HIV, hepatitis C, and cancer. Even more exciting is the potential of RNAi in agriculture, RNAi has provided a way to control pests and diseases, introduce novel plant traits and increase crop yield. The technology became a powerful tool to understand the functions of individual genes and also proved useful for molecular breeders to produce improved crop varieties. Using RNAi, scientists have developed novel crops such as nicotine-free tobacco, non-allergenic peanuts, decaffeinated coffee, and nutrient fortified maize among many others. For science in general it is already a tool of large scale reverse genetic approaches and aids in unravelling gene functions in many species.

Small, non-coding RNAs are important regulators of posttranscriptional gene silencing in eukaryotes. Studies in Arabidopsis thaliana indicated a complex set of small RNAs deriving from distinct doublestranded RNA (dsRNA) precursors, which differ with respect to their origin and subsequent processing. Based on their biogenesis and function plant small RNAs are classified as repeated-associated small interfering RNAs (ra-siRNAs), trans-acting siRNAs (ta-siRNAs), naturalantisense transcript-derived siRNAs (nat-siRNAs) and microRNAs (miRNAs). Cloning approaches and computational predictions led to the identification of miRNAs from diverse plant species. Interestingly, particular miRNA families and their corresponding targets were found to be conserved in distantly related plant species suggesting a common origin of miRNA-regulated pathways in plants. While miRNAs were first described in 1993 [2], it is only in the last five years or so thattheir significance has been recognised. In humans, approximately $50 \%$ of genomic DNA is transcribed in RNA with $2 \%$ of this translated into protein and $98 \%$ non-coding [3]. It is currently estimated that miRNA genes constitute about $2 \%$ of the known genes, but that in eukaryotes more than $60 \%$ of genes may be regulated by miRNAs. Thousands of miRNAs have now been identified in various organisms (www.mirbase. org).

Among the exciting applications in which RNA-mediated impact in agriculture is the improvement of essential food crops, a number of successful applications have already emerged, particularly the ability of
RNAi to confer resistance to common plant viruses and fortification of plants such as tomatoes with dietary antioxidants. Because most RNAi research has been carried out in Arabidopsis, there are substantial gaps inour knowledge about the RNAi mechanisms at work in all of the economically important crops. For example, the parallel RNAi silencing pathways for tasiRNA, and natsiRNA were described in Arabidopsis have not been clearly elucidated in most crop species.

It became obvious that there are many opportunities for RNA-mediated crop improvement; for example, alteration in plant architecture and It was shown that OsmiR156 play a big role in ideal plant architecture in rice [4], biotic and abiotic stress responses; changing environmental condition will be necessary to feed ever-increasing population and recently, several small RNAs have been identified under biotic and abiotic stress conditions [5], nutritional improvement; synthesis and optimization of various key nutrients iron, carotenoids, flavonoids, antioxidants, vitamins, fatty acid, and amino acid composition in cereals, fruits, etc. to develop bio-fortified crops, increase in shelf-life of fruits and vegetable to minimize senescence and deterioration of fruit quality and post-harvest spoilage during transportation and storage. The study of small RNAs can be introduced to identify the role that these small molecules in the shelf-life of fruits, also enhancement of secondary metabolites; the RNA ican be used to facilitate synthesis and production of commercially valuable plant-derived drugs, fragrances, pigments, flavors, and volatile oils, and one more thing for RNA-mediated crop improvementis the seedless of fruit development, and this application are appreciated by the consumers for fresh consumption as well as processed fruit products.

The study of RNAi has become increasingly more rewarding and continually expanding following the completion of several recent genome sequencing projects. Manipulating new RNAi pathways, which generate small RNA molecules to amend gene expression in crops, can produce new quality traits and having better potentiality of protection against abiotic and biotic stresses. Furthermore, conserved miRNAs can be analysed in crop plants; this will provide the possibility to exploit the potential of endogenous miRNAs for the improvement of quality traits in crop plants. Also, the artificial microRNA (amiRNA)-mediated approach should have broad applicability for engineering multiple stress-responsive genes in crop plants [6]. Current agricultural technology needs more and more molecular tools to reduce current crop loss and feed extra mouths, which according to a recent estimate by the FAO (Food and Agriculture Organization) will increase by two billion over the next 30 years. The RNA-mediated crop improvement, mentioned

*Corresponding author: Basel Khraiwesh, Plant Stress Genomics Research Center, King Abdullah University of Science and Technology, Thuwal 23955-6900, Kingdom of Saudi Arabia, E-mail: basel.khraiwesh@kaust.edu.sa

Received October 17, 2011; Accepted October 18, 2011; Published October 19 2011

Citation: Khraiwesh B (2011) RNA-Mediated Crop Improvement. J Biotechnol Biomaterial 1:107e. doi:10.4172/2155-952X.1000107e

Copyright: (c) 2011 Khraiwesh B. This is an open-access article distributed under the terms of the Creative Commons Attribution License, which permits unrestricted use, distribution, and reproduction in any medium, provided the original author and source are credited. 
above, describes one such powerful innovation. Hopefully, the technology that has been developed by the scientists from developed countries will be available to any lab including those in the developing countries, where work utilizing RNAi technology is either in progress or going to be launched shortly. The technology is well developed and can be applied directly to evolve a crop resistant to stresses. Since this technology offers a great potential in understanding gene functions and utilize them to improve crop quality and production, it is a matter of time before we see the products of this RNAi research in the farmers' fields around the world.

\section{References}

1. Khraiwesh B, Arif MA, Seumel GI, Ossowski S, et al. (2010) Transcriptional control of gene expression by microRNAs. Cell 140: 111-122.
2. Lee RC, Feinbaum RL, Ambros V (1993) The C. elegans heterochronic gene lin-4 encodes small RNAs with antisense complementarity to lin-14. Cell 75 843-854.

3. Zhang C (2008) MicroRNomics: a newly emerging approach for disease biology. Physiological Genomics 33: 139-147.

4. Jiao Y, Wang Y, Xue D, Wang J, Yan M, et al. (2010) Regulation of OsSPL14 by OsmiR156 defines ideal plant architecture in rice. Nature Genetics 42: 541 544

5. Khraiwesh B, J-K Zhu, Zhu J (2011) Role of miRNAs and siRNAs in biotic and abiotic stress responses of plants. Biochim Biophys Acta. (BBA)-Gene Regulatory Mechanisms.

6. Khraiwesh B, Ossowski S, Weigel D, Reski R, Frank W (2008) Specific Gene Silencing by Artificial MicroRNAs in Physcomitrella patens: An Alternative to Targeted Gene Knockouts. Plant Physiology 148: 684-693.

- User friendly/feasible website-translation of your paper to 50 world's leading languages 